\title{
Nominación de algunos números fraccionarios en miskito
}

\section{Victor Winsin Castrillo}

\section{INTRODUCCION}

Desde su apertura, la Educación Intercultural Bilingue (EIB) ha hecho esfuerzos por capacitar a una buena cantidad de docentes, especialmente a los del sector urbano y algunos de los centros rurales cercanos. En este sentido son incalculables los aportes que han hecho estos docentes al Sistema Educativo Autonómico Regional (SEAR), sin embargo, aún existen grandes vacíos en el currículo del programa de Educación Intercultural Bilingüe, especialmente con respecto a los textos de matemáticas.

Enseñar en lengua miskita los contenidos de matemáticas es realmente una ardua labor, especialmente en lo referente al vocabulario técnico matemático. En el caso de los nombres de los números fraccionarios es toda una hazaña, puesto que los docentes y estudiantes del programa de Educacion Intercultural Bilingüe no cuentan con un documento que les enseñe estos números en lengua miskita.

Este estudio nos brinda algunos nombres de números fraccionarios en lengua miskita, algo poco abordado en el Subsistema Educativo Autonómico Regional, asi como algunos obstáculos a nivel del proceso de enseñanzaaprendizaje de los alumnos y alumnas que cursan el 5to y el 6to grado de Educacion Intercultural Bilingüe Miskito-Español' .

Con el fin de fortalecer la enseñanza-aprendizaje de los nombres de algunos números fraccionarios en la lengua miskitu, nos planteamos: evaluar el uso de los nombres de los números fraccionarios en esta lengua, analizar las dificultades del uso de los nombres de los números fraccionarios en esta misma lengua y elaborar un listado sencillo con los nombres de los números fraccionarios básicos en miskito. Todo esto, a través de entrevistas directas y observación participativa con comunitarios, líderes comunales y docentes, asi como con el aporte de fuentes bibliográficas.
Este estudio es una contribución para el fortalecimiento de la Educación Intercultural Bilingüe (EIB) y de la cultura indígena miskitu, con la participación de todos los actores y contrapartes de la investigación y del sector educativo. Pretendemos retribuir a la comunidad de estudio la información recopilada, a través de ejemplares del informe final, tanto a los líderes como al personal educativo de la escuela "SimeónWalls", que fue sujeto de esta investigación, seguido de una presentación y capacitación de los resultados finales. También se retribuirá cinco ejemplares a las contrapartes del fondo.

\section{La comunidad de Kuamwatla}

Kuamwatla es una comunidad perteneciente al territorio Prinzu Auhyam, en el municipio de Prinzapolka. Su nombre proviene del miskito, que traducido al español significa "hábitat de pavos". Es considerada una de las primeras comunidades indígenas miskitas de esa región. Está ubicada en la franja fronteriza de la Región Autónoma Atlántico Norte (RAAN) y de la Región Autónoma Atlántico Sur (RAAS). Su cabecera regional es Puerto Cabezas y su cabecera municipal es Alamikangbam. Limita al Este con el Mar Caribe; al Oeste con el Llano; al Norte con la comunidad de Prinzapolka, y al Sur con la comunidad de Sheran, en Sandy Bay Sirpy.

La comunidad está asentada en una franja de tierra llana, su nivel aproximado es de cinco metros de altura sobre el nivel del mar, frente a la pequeña laguna que lleva el mismo nombre de la comunidad. La única vía de acceso a la comunidad es acuática, a través de la barra de Prinzapolka. Luego de esta entrada se toma el desvio del río Kwambila. Se logra alcanzar la comunidad en ocho horas usando un motor fuera de borda de 75 caballo y, si es Ponking, en un lapso de doce horas desde Puerto Cabezas.

Según las historias orales transmitidas de generacion en generacion, los primeros pobladores se establecieron en esta comunidad debido a que eran cazadores de animales silvestres, y durante su trayecto por estos

\footnotetext{
I El estudio se hizo en la comunidad de Kwamwatla, que describiremos adelante, en la escuela primaria Simeon Walls.
} 
parajes encontraron abundancia de pavos, por lo que decidieron establecerse allí dedicándose también a la recolección de frutas silvestres. No se sabe con exactitud quiénes fueron los que llegaron primero y cuándo se establecieron.

\section{Obstáculos para la nominación de los números fraccionario en miskitu}

Aquí solamente trataremos de entender el porqué los docentes y los estudiantes no están aprendiendo los nombres de los números fraccionarios en miskito.

Según el MECD (2003:1099), las fracciones se representan de esta manera:
a. $1 / 4 \mathrm{kum}$
b. $2 / 8 \mathrm{kum}$
g. $4 / 16 \mathrm{kum}$
h. $8 / 32$ kum

Podemos ver que, igual que en el texto Kulki Sakaia Bukka $4^{a}$, en el SEAR (2010:100-120) sólo aparecen los simbolos y no dice cómo se expresa verbalmente la fracción en miskito. En ambos se repite el número "kum" (uno/ un), pero se deja implicito el equivalente miskito de (a) 'cuarto', (b) 'octavo', (g) 'dieciseisavo' y (h) 'treintidosavo y doceavo'. Lo triste de esto es que éste sería el momento oportuno para que el alumno aprendiera la manera de expresar verbalmente las fracciones en miskito. Es aquí donde se encuentra el gran vacío en torno a la nominación fraccionaria en lengua materna.

\section{La nominación fraccionaria en miskitu}

Algunos documentos medianamente nos aclaran sobre la nominación de números fraccionarios en miskito. Pero los ancianos, en las comunidades manejan otra forma de enunciarla, sin embargo, lo esencial de este asunto es que no existe un documento que recoja y aclare esto mediante estudios concienzudos, sino que se maneja a conveniencia del que lo enuncia. Por ejemplo, la Sociedad Bíblica de Nicaragua, en el transcurso del tiempo tiene diversas nominaciones para las mismas fracciones:

Según la Sociedad Bíblica de Nicaragua (1974:168, 531):

1/2 Bakriki 1/3 Manka Yumpa

Según la Sociedad Bíblica de Nicaragua (1999: 375):

1/2: Bakriki; 1/3: Yuhmpa Wina Kumi

Según la Sociedad Bíblica de Nicaragua 2010: 439):

1/2: Bakriki; 1/3: Manka Yumhpa Wina Kumi

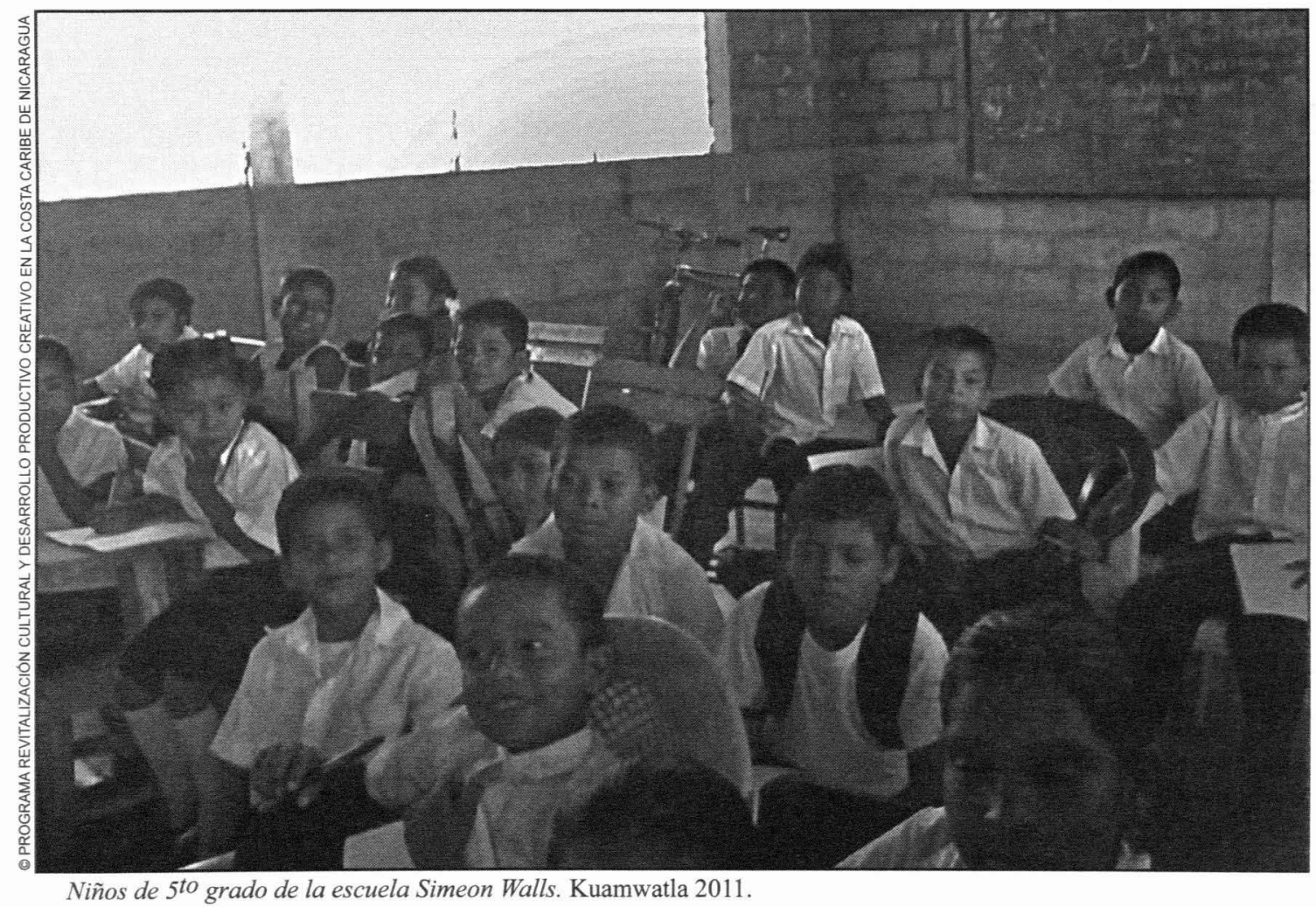




\section{RESULTADOS Y DISCUSION}

Hemos abordado el grado de utilización de los números fraccionarios en miskito. Para esto se ha tomado como refrencia el 5 to y 6 to grado de la escuela Simeón Walls de la comuniadad de Kuamwatla. Se tiene previo conocimiento que la utilización de los números fraccionario en lengua miskita, sobre todo en los textos escolares, es limitado, pues se cuenta únicamente con los símbolos de los números fraccionarios $(1 / 2,1 / 3$, etcétera) y el enunciado $\mathrm{kum}$ que indica, para cada caso, un (x)avo, donde $\mathrm{x}$ es el número de partes, pero no se dan los equivalentes en miskito de palabras como 'octavo', 'dieciseisavo -o medio, tercio, cuarto, quinto, sexto, septimo...etcétera. Es decir, que el número de partes o partitivo se deja implícito, como si no se supiera cómo se expresa este tipo de numeral en miskito. Como nuestro objetivo para este caso es dejar claro si se están utilizando o no de alguna manera los números fraccionarios en miskito por parte de los alumnos y maestros, los resultados que arrojaron las encuestas fueron los siguientes:

\section{Utilización de los números fraccionarios en miskitu}

Las encuestas hechas entre los alumnos y docentes en torno a la utilización de los números fraccionarios en miskito dieron los siguientes resultados:

\section{Alumnos}

¿Utilizan en sus grados los números fraccionarios en miskito?

\begin{tabular}{|c|c|}
\hline Si lo utilizan & No lo utilizan \\
\hline $0 \%$ & $100 \%$ \\
\hline
\end{tabular}

\section{Docentes}

¿Imparten en sus colegios los números fraccionarios en miskito?

\begin{tabular}{|c|c|}
\hline Si se imparte & No se imparte \\
\hline $0 \%$ & $100 \%$ \\
\hline
\end{tabular}

A través de la observación directa de la clase de matemática y según el diagnóstico de los alumno(as) y docentes se comprobó que, tanto los alumnos como los docentes de 5 to y 6 to grado de la escuela Simeón Walls, no utilizan los nombres de los números fraccionarios en el idioma miskito. El $100 \%$ de los consultados, tanto alumnos como docentes, respondieron que no los utilizan en miskito.

\section{Conocimiento sobre los números fraccionarios}

Con el fin de indagar más acerca del tema de esta investigación se decidió ahondar acerca del conocimiento o el grado de dominio por parte de profesores y alumnos sobre los números fraccionarios en miskito. En este sentido, a estudiantes, docentes, técnicos y expertos se les formuló la pregunta sobre si conocian de su utilización en algún documento.

¿Conoces algún documento que hable sobre los números fraccionarios en miskitu?

\begin{tabular}{|l|c|c|c|}
\hline & $\begin{array}{c}\text { Matemática } \\
5^{\circ} \text { y 6 } \\
(\text { MECD 2003) }\end{array}$ & $\begin{array}{c}\text { Nuevo } \\
\text { Testamento } \\
\text { en miskito }\end{array}$ & $\begin{array}{c}\text { Matemática } \\
\text { de 4to grado } \\
\text { SEAR (2010) }\end{array}$ \\
\hline Estudiantes & $\mathbf{1 0 0 \%}$ & $\mathbf{0} \%$ & $\mathbf{0} \%$ \\
\hline Docentes & $\mathbf{1 0 0 \%}$ & $\mathbf{0} \%$ & $\mathbf{0} \%$ \\
\hline Técnicos & $\mathbf{1 0 0} \%$ & $\mathbf{0} \%$ & $\mathbf{1 0 0} \%$ \\
\hline Expertos & $\mathbf{1 0 0} \%$ & $\mathbf{0} \%$ & $\mathbf{1 0 0} \%$ \\
\hline
\end{tabular}

Y según entrevistas con los alumnos y docentes, solo conocen el texto de matemáticas de 5 to y 6 to grado del MECD, (2003) el cual contiene el conjunto de números fraccionarios donde únicamente aparecen los símbolos.

En entrevistas hechas al técnico del MINED municipal de Prinzapolka Elmer Lago, al técnico departamental del MINED Orbin Borge, y a los expertos en Educación Intercultural Bilingüe Adistan Bons y Donald Conrado, éstos mencionaron la primera traducción del Nuevo Testamento en miskito, de la Sociedad Bíblica de Nicaragua (1974). Este libro tiene en sus versículos indicios de los números fraccionarios en miskito, así también el texto de Matemáticas de 5to y 6to grado del MECD (2003) donde sólo aparecen algunos símbolos. También mencionaron al SEAR (2010) con el texto de Matemáticas de 4to grado. Esto muestra que no hay documento claro con nombres de números fraccionarios en lengua miskita.

Sin embargo, consultando a los ancianos(as) de la comunidad de Kuamwatla, como Gonzalo Gómez, Eludia 
Wade, José Zelaya y Mouphy Flores, sobre ejemplos de números fraccionarios en miskito, ellos nos brindaron algunos aportes al respecto.

\section{Obstáculos del uso de los números fraccionarios en miskito}

Entre los principales obstáculos para la articulación y utilización de los números fraccionarios en miskitu logramos indentificar los cuatro más importantes:

El primero es el que hemos estado denunciando a través de todo este artículo: que en los textos de matemáticas de 5 to y 6 to grado en miskito, solo están plasmados los símbolos de los números fraccionarios y no su nominación. Esto podemos verificarlo en el texto del MECD (2003:1099):
a. $1 / 4 \mathrm{kum}$
b. $2 / 8$ kum
g. $4 / 16 \mathrm{kum}$
h. $8 / 32$ kum

El segundo obstáculo se puso de manifiesto luego de consultar al docente de 5to. Grado Josue Emilio Taylor Castellon y al profesor de 6to. Grado Emos Benneth Jacobo. Y es que no se conoce ningun documento que contengan los nombres de los números fraccionarios en el idioma miskito. Es decir, no se cuenta con materiales pedagógicos ni didácticos que abarquen el estudio de los números fraccionarios en miskito.

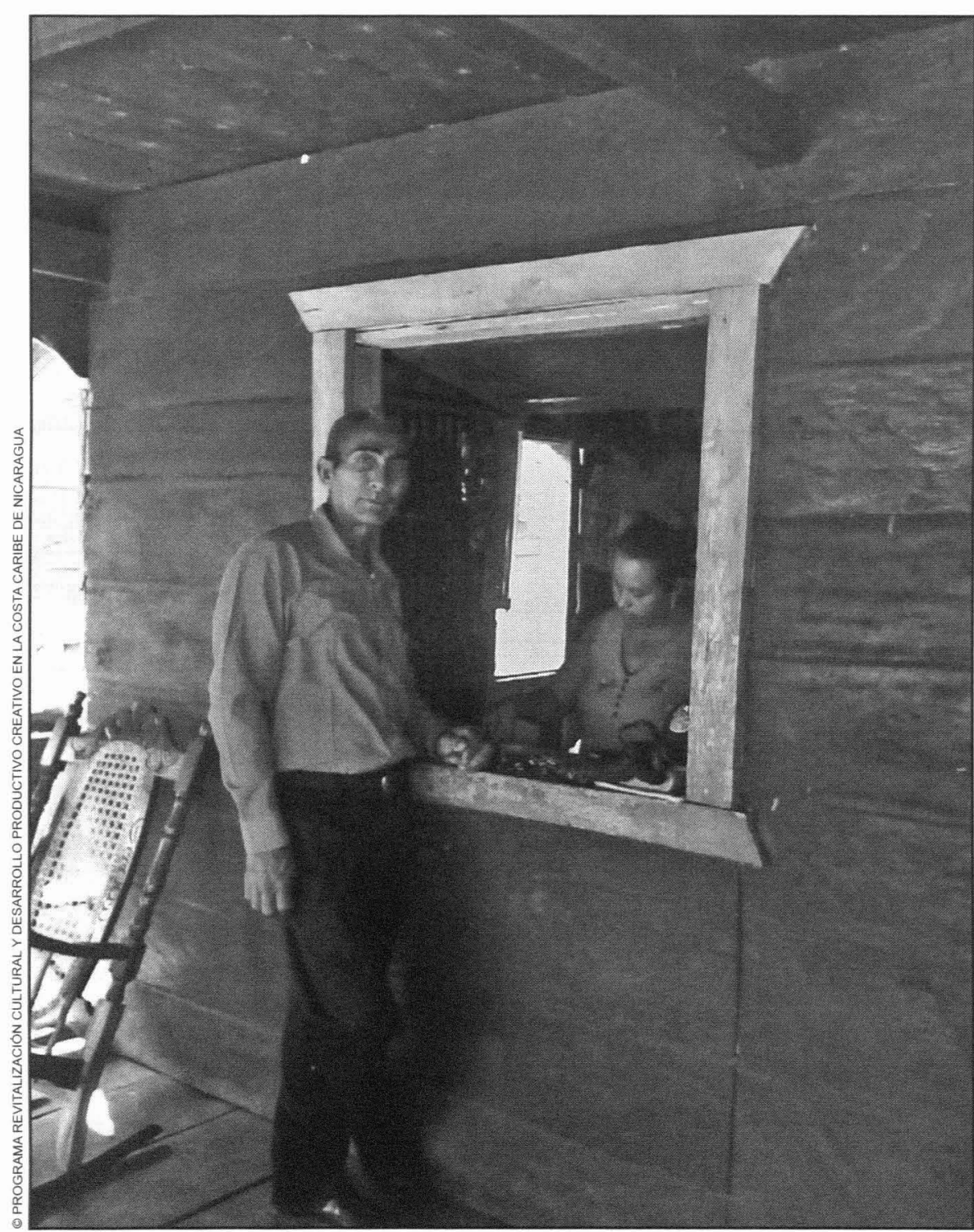

Profesor haciendo entrega de un informe investigativo. Kuamwatla 2011.
El tercer abstáculo se identificó gracias principalmente a las entrevistas con los docentes de 5to y 6to grado, Gardo Josué Emilio Taylor Castellon y Emos Benneth Jacobo, respectivamente. Ellos manifiestan que no han tenido capacitación sobre los nombres de los números fraccionarios en el idioma miskito, tema de suma importancia para incorporarlo al plan de estudios de 5to y 6 to grados.

El cuarto obstáculo que existe para que no sea impartida la clase de números fraccionarios en miskitos es que los docentes de eta escuela en Maniwatla, a pesar de ser maestros normalistas graduados en la modalidad monolingüe castellano, no están especializados en Educacion Intercultural Bilingüe y estan enseñando empiricamente en esta modalidad. 


\section{Listado ilustrativo de algunos números fraccionarios en miskito}

A continuación presentamos una lista básica de números fraccionarios, que puede aportar al conocimiento de los números fraccionarios en miskito, según los ancianos, la Sociedad Bíblica de Nicaragua (1974), la Sociedad Bíblica de Nicaragua (1999), la Sociedad Bíblica de Nicaragua (2010) y los técnicos consultados.

\begin{tabular}{|c|c|c|c|}
\hline$N^{\circ}$ & Nominación & Gráfica & Descripción \\
\hline $1 / 2$ & $\begin{array}{l}\text { Baikanka wal wina kum } \\
\text { (según los ancianos) }\end{array}$ & & $\begin{array}{l}1 / 2 \text { de la piña tiene } \\
\text { hoja }\end{array}$ \\
\hline $1 / 3$ & $\begin{array}{l}\text { Baikanka yumpa wina kum } \\
\text { (según la Sociedad Biblica) }\end{array}$ & & $\begin{array}{l}1 / 3 \text { de banano esta } \\
\text { separado }\end{array}$ \\
\hline $1 / 4$ & $\begin{array}{l}\text { Baikanka walwal wina kum } \\
\text { (según los ancianos) }\end{array}$ & & $\begin{array}{l}1 / 4 \text { de la piña estan } \\
\text { separando }\end{array}$ \\
\hline $1 / 5$ & Baikanka mat wina kum & & $\begin{array}{l}1 / 5 \text { del chocolate } \\
\text { es aris }\end{array}$ \\
\hline $1 / 10$ & Matwal wina kum & & $1 / 10$ del chocolate \\
\hline $2 / 2$ & $\begin{array}{l}\text { Baikanka wal wina wal } \\
\text { (según los técnicos) }\end{array}$ & & $\begin{array}{l}\text { Los } 2 / 2 \text { del banano } \\
\text { lo tiene a mano }\end{array}$ \\
\hline $2 / 3$ & $\begin{array}{l}\text { Baikanka yumpa wina wal } \\
\text { (según los técnicos) }\end{array}$ & & $\begin{array}{l}2 / 3 \text { de la } \\
\text { guanabana no está } \\
\text { dividido }\end{array}$ \\
\hline $2 / 4$ & $\begin{array}{l}\text { Baikanka walwal wina wal } \\
\text { (según los técnicos) }\end{array}$ & & $\begin{array}{l}2 / 4 \text { de la guayaba } \\
\text { tiene forma de } \\
\text { triangulo }\end{array}$ \\
\hline
\end{tabular}




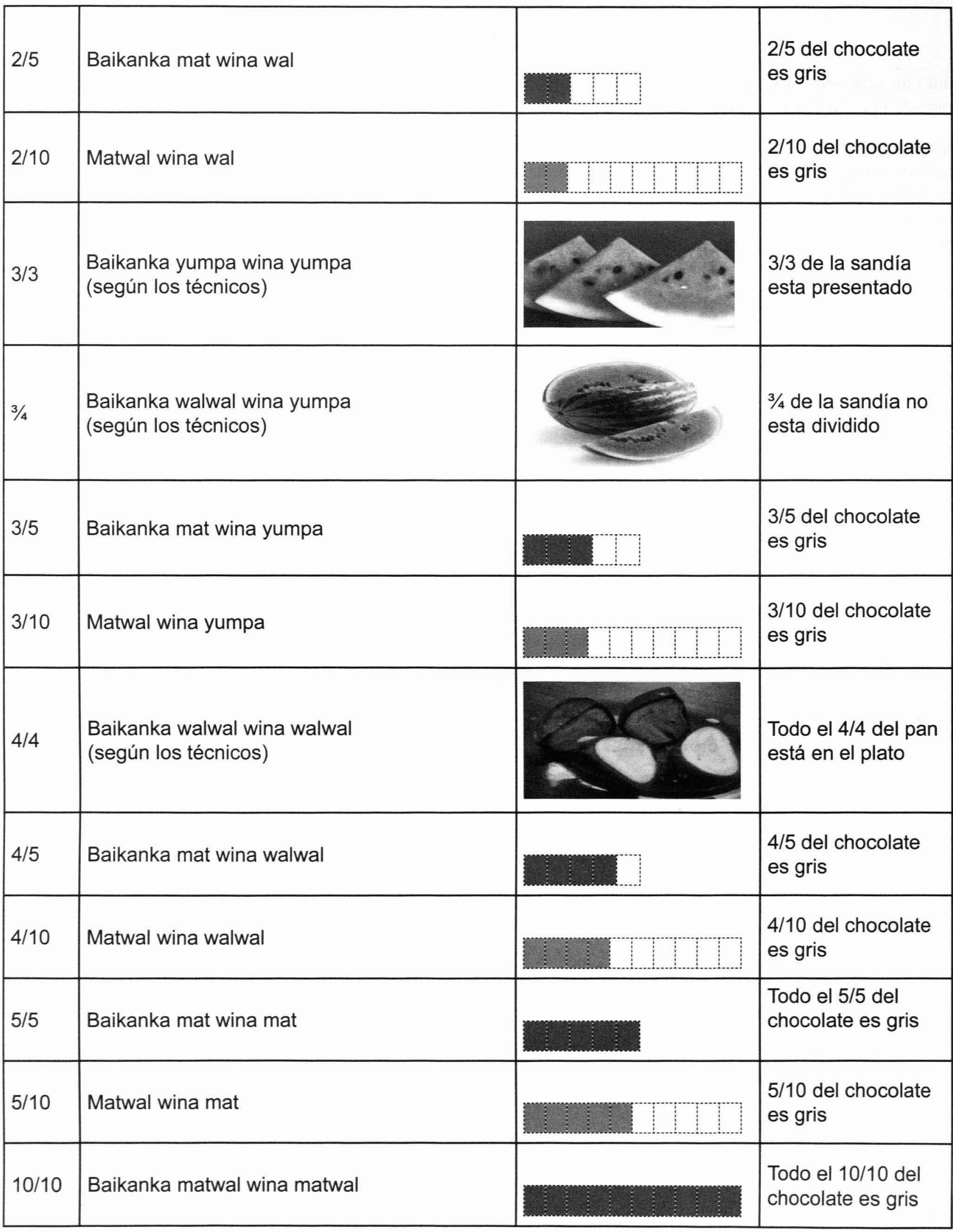




\section{CONCLUSIONES}

Tanto los educandos y docentes no utilizan los nombres de los números fraccionarios en el idioma miskitu, en 5to y 6to grado de la escuela Simeón Walls.

Las principales dificultades para el uso de los nombres de los números fraccionarios en el idioma miskito, en 5to y 6to grado de la escuela Simeón Walls son: en los textos de matemática de 4to, 5to y 6to grado en lengua miskita, solo existen los símbolos de los números fraccionarios y no su nominación; no existen documentos que contengan explícitamente los nombres de los números fraccionarios en la lengua miskita; los docentes no reciben capacitación del MINED-SEAR sobre los nombres de los números fraccionarios en la lengua miskita, y estos docentes, aunque son maestros normalistas en la modalidad monolingüe castellano, no son especialista en Educacion Intercultural Bilingüe, es decir, que se están desempeñando empíricamente en esta modalidad.

\section{RECOMENDACIONES}

A la Secretaria de Educación del Gobierno Regional Autónomo:

- Crear una Academia de Matemáticas y de otras disciplinas según la misión y visión del Subsistema Educativo Autonómico Regional.

A la Dirección de Educación Intercultural Bilingüe:

- Tomar en cuenta los resultados y sugerencias de este estudio, para hacer factible una enseñanza-aprendizaje de los números fraccionarios en miskito.

- Escribir en los textos de matemática de 4 to, 5to y 6 to grado todos los nombres de los números fraccionarios en miskito.

- Seleccionar personal idóneo en la elaboración de las bibliografías de Matemáticas.

A las universidades:

- Brindar acompañamiento a la Secretaria de Educación de los Gobiernos de las Regiones Autónomas, para crear una academia de matemáticas y de otras disciplinas científicas.

- Dar acompañamiento a los proyectos de elaboración de textos de matemáticas en miskito dentro de sus posibilidades.

A los docentes:

- Enseñar a los educandos de 4to, 5to y 6to grados, los nombres de los números fraccionarios en miskito.

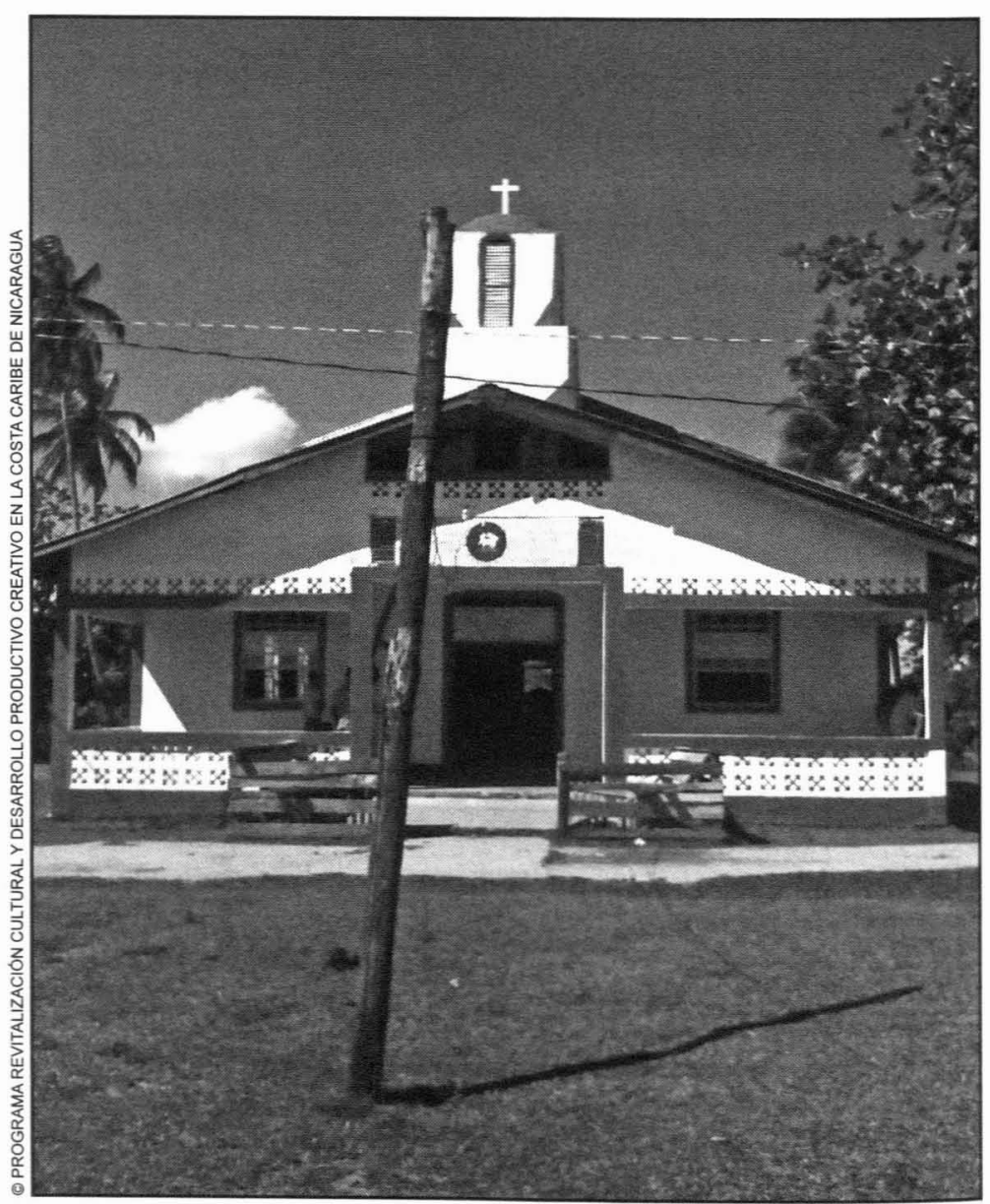

La histórica iglesia Morava fundada en 1971. Kuamwatla 2011. 


\section{REFERENCIAS BIBLIOGRAFICAS}

Instituto de Promoción e Investigaciones Lingüísticas y Rescate Cultural (IPILC). (2003). Sistema Educativo Autonómico Regional. Por la unidad en la diversidad Costa Caribe nicaragüense. Managua: URACCAN.

Matus, R. (2004). Nuestra Ortografia. Managua: Matus Lazo Ediciones.

Nicaragua. Ministerio de Educación. (MED). (1989). Matemática 5: Quinto grado. Managua: MED.

Nicaragua. Ministerio de Educación Cultura y Deporte (MECD). (2000). Matemática 5. (Versión Miskitu).

OCEANO. (2000). Enciclopédia Didática de Matemáticas. Barcelona: Oceano Group

Real Academia Española \& Asociación de Academias de la Lengua Española (2006). Diccionario esencial de la lengua española. Madrid: Espasa Calpe.

Romero V., G. (1996). Historia de la Costa Atlántica. Managua: UCA/ Talleres Gráficos.

Sistema Educativo Autonómico Regional (SEAR). (2010). Kulki Sakaia Sinska 4a $=$ =Matemática 4. Managua: MECD.

United Bible Societies. (1974). Dawan bila: la raya waungkataya, naiwa yua uplika nani dukiara=El nuevo testamento en miskito. [s.1.]: Sociedades Bíblicas Unidas.

United Bible Societies. (1999). Dawan Bila Aiska. [s.l.]: Sociedades Bíblicas Unidas. 